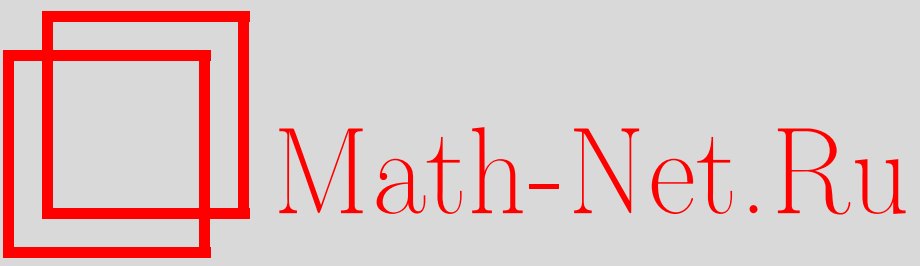

А. А. Ордин, Обобщенное барицентрическое разбиение треугольника и полугруппы мебиусовых преобразований, УМН, 2000, том 55, выпуск 3, 185-186

DOI: https://doi.org/10.4213/rm304

Использование Общероссийского математического портала Math-Net.Ru подразумевает, что вы прочитали и согласны с пользовательским соглашением

http://www.mathnet.ru/rus/agreement

Параметры загрузки:

IP: 52.23 .180 .231

26 апреля 2023 г., $17: 32: 59$ 


\title{
ОБОБЩЕННОЕ БАРИЦЕНТРИЧЕСКОЕ РАЗБИЕНИЕ ТРЕУГОЛЬНИКА И ПОЛУГРУППЫ МЕБИУСОВЫХ ПРЕОБРАЗОВАНИЙ
}

\author{
А.А. Ордин
}

Данный треугольник разрезаем медианами на 6 треугольников, каждый из которых в свою очередь разрезаем медианами на 6 меньших треугольников, и т. д. до бесконечности. Следующий вопрос принадлежит В. Стаховскому: найдутся ли для произвольного заданного треугольника сколь угодно близкие к нему с точностью до подобия треугольники из построенного множества? И. Барани и А. Бердон в [1] ответили на этот вопрос положительно. В этой работе мы развиваем идею их доказательства для случая разбиения треугольника чевианами, проходящими через точку с фиксированными барицентрическими координатами относительно вершин треугольника.

Зафиксируем числа $p=\left(p_{0}, p_{1}, p_{2}\right)>0, p_{0}+p_{1}+p_{2}=1$. Пусть $\mathbf{A}=\left(A_{0}, A_{1}, A_{2}\right)$-треугольник, $C=p_{0} A_{0}+p_{1} A_{1}+p_{2} A_{2}$. Рассмотрим 6 треугольников, на которые треугольник $\mathbf{A}$ разбивается прямыми $A_{i} C$. Занумеруем вершины каждого из них индексами $0,1,2$ так, чтобы вершина, совпадающая с точкой $A_{i}$, получила номер $i$ и чтобы получающаяся ориентация совпадала с ориентацией треугольника $\mathbf{A}$. Построенный набор треугольников мы обозначим через $B(\mathbf{A})$. Пусть $B^{0}(\mathbf{A})=\{\mathbf{A}\}, B^{n}(\mathbf{A})=\bigcup_{\mathbf{A}^{\prime} \in B^{n-1}(\mathbf{A})} B\left(\mathbf{A}^{\prime}\right), n \in \mathbb{N}, T(\mathbf{A})=\bigcup_{n=0}^{\infty} B^{n}(\mathbf{A})$.

Теорема. Для любого треугольника $\mathbf{B}$ в T(A) найдется треугольник, сколь угодно близкий к $\mathbf{B}$ с точностью до подобия.

ПЛАН ДОКАЗАТЕЛЬСТВА. Без ограничения общности мы можем считать, что $p_{0} \leqslant p_{1} \leqslant p_{2}$. Треугольнику $\mathbf{A}=\left(A_{0}, A_{1}, A_{2}\right)$ сопоставим вектор $l(\mathbf{A})=\left(\left|A_{1}-A_{2}\right|^{2},\left|A_{2}-A_{0}\right|^{2},\left|A_{0}-A_{1}\right|^{2}\right)$. Обозначим через $\mathbf{A}^{i j}, i, j \in \overline{0,2}, i \neq j$, треугольник из $B(\mathbf{A})$, прилегающий к стороне, противоположной $A_{i}$, и к вершине, противоположной в этой стороне к $A_{j}$. Тогда имеем для любой перестановки $i, j, k$ номеров $0,1,2([1]): l\left(\mathbf{A}^{i j}\right)=F_{i j} l(\mathbf{A}), F_{i j}=M_{i j}^{-1} U_{p_{i}} V_{p_{j} /\left(1-p_{i}\right)} M_{i j}$, где $U_{\alpha}=\left(\begin{array}{ccc}1 & 0 & 0 \\ 1-\alpha & \alpha & -\alpha(1-\alpha) \\ 0 & 0 & \alpha^{2}\end{array}\right), V_{\alpha}=\left(\begin{array}{ccc}\alpha^{2} & 0 & 0 \\ 0 & 1 & 0 \\ -\alpha(1-\alpha) & 1-\alpha & \alpha\end{array}\right), M_{i j}\left(\begin{array}{l}a_{0} \\ a_{1} \\ a_{2}\end{array}\right)=\left(\begin{array}{c}a_{i} \\ a_{j} \\ a_{k}\end{array}\right)$.

Ясно, что множество векторов треугольников из $T(\mathbf{A})$ есть множество образов (орбита) $l(\mathbf{A})$ под действием полугруппы, порожденной операторами $F_{i j}$.

Лемма 1 ([1]). $a=\left(a_{0}, a_{1}, a_{2}\right)$ есть вектор некоторого треугольника тогда и только тогда, когда $a_{i}>0, \forall i=0,1,2$ u $q(a)=a_{0}^{2}+a_{1}^{2}+a_{2}^{2}-2 a_{0} a_{1}-2 a_{1} a_{2}-2 a_{2} a_{0}<0$. Множество таких векторов есть открытый круговой конус $C$ с осью $L=\{t(1,1,1): t \in \mathbb{R}\}$, вписанный в положительньй октант $\mathbb{R}^{3}$. Число -q(a)/16 есть квадрат площади треугольника с квадратами сторон $a_{0}, a_{1}, a_{2}$.

Операторы $F_{i j}$ отображают конус $C$ на себя, поэтому мы можем рассматривать их как мебиусовы преобразования $g_{i j}$ единичного круга $\Delta=\{z:|z|<1\}$ ([1]). Пусть $G$ - порожденная ими полугруппа. Нам достаточно доказать, что $G$ всюду плотна в группе $S \Gamma$ всех собственных мебиусовых преобразований $\Delta$. Пусть $w=e^{\pi i / 3}, z_{0}=1, z_{1}=w^{2}, z_{2}=w^{4}, M \subset \Delta$-гиперболический треугольник с вершинами $z_{0}, z_{1}, z_{2}$. Полуплоскость из $\Delta \backslash M$ напротив точки $z_{i}$ мы обозначаем через $\Omega_{i}$. Через $\bar{\Omega}$ мы обозначаем замыкание подмножества $\Omega \subset \Delta$ в $\Delta$.

Лемма 2. Пусть $H \subset \Gamma-$ полугруппа, $P \subset \Delta-$ многоугольник с конечным числом вериин на абсолюте $\left\{v_{1}, \ldots, v_{k}\right\}$ и всякая орбита $H \cdot z$ пересекается $с$ Р. Пусть для всякого $i \in \overline{1, k}$ существует гиперболическая изометрия $g_{i} \in H$, для которой $v_{i}$ есть отталкивающая неподвижная точка. Тогда существует компакт $K \subset \Delta$, с которым пересекается всякая орбита $H \cdot z$. 
Лемма 3. Пусть $H \subset \Gamma-$ полугруппа и существует компакт $K \subset \Delta$ такой, что для всякой точки $z \in \Delta H \cdot z \cap K \neq \varnothing$. Тогда замыкание полугруппы $\bar{H}$ является группой.

Леммы 2 и 3 применяются для доказательства того, что $\bar{G}$ есть группа. Пару номеров $(i, j)$, $i, j \in \overline{0,2}, i \neq j$, назовем регулярной, если $p_{j}>p_{i}\left(1-p_{i}\right)$, нерегулярной, если $p_{j} \leqslant p_{i}\left(1-p_{i}\right)$, нейтральной, если $p_{j}=p_{i}\left(1-p_{i}\right)$, и строго нерегулярной, если $p_{j}<p_{i}\left(1-p_{i}\right)$.

Лемма 4. Пусть $i, j, k$ - произвольная перестановка номеров $0,1,2$. Eсли $(i, j)-$ регулярная пара, то $g_{i j}$ - гиперболическая изометрия с отталкивающей неподвижной точкой $z_{i}$ и $A_{g_{i j}} \subset \Omega_{j}$. Если $(i, j)$ - нейтральная пара, то $g_{i j}$ - параболическая изометрия с неподвижной точкой $z_{i}$. Если $(i, j)$ - строго нерегулярная пара, то $g_{i j}-$ гиперболическая изометрия с притягивающей неподвижной точкой $z_{i}$ u $A_{g_{i j}} \subset \Omega_{k}$. Кроме того, $g_{i j}\left(\overline{\Omega_{k}}\right) \cap \overline{\Omega_{j}}=\varnothing, g_{i j}\left(\Omega_{j}\right) \subset \Omega_{j}$.

Случай 1 : среди пар $(1,0),(2,0),(2,1)$ есть регулярные. Тогда найдутся номера $i, j$ такие, что пары $(i, j)$ и $(j, i)$ регулярны. Пусть $P_{1}=\left(g_{i j}\left(\Omega_{k}\right) \cup g_{j i}\left(\Omega_{k}\right)\right) \cap M$.

Лемма 5. В случае 1 любая орбита $G \cdot z$ содержит точки из $P_{1}$.

В силу леммы 4 многоугольник $P_{1}$ имеет две вершины $z_{i}, z_{j}$ на абсолюте, причем $z_{i}$ есть отталкивающая неподвижная точка $g_{i j}, z_{j}$ есть отталкиваюшая неподвижная точка $g_{j i}$.

Случай 2: пары $(1,0),(2,0),(2,1)$ нерегулярны. Пусть $P_{2}=\left(g_{20}\left(\Omega_{2}\right) \cup g_{21}\left(\Omega_{2}\right)\right) \cap M$.

Лемма 6. В случае 2 любая орбита $G \cdot z$ содержит точки из $P_{2}$.

В силу леммы 4 многоугольник $P_{2}$ имеет две вершины $z_{0}, z_{1}$ на абсолюте, причем $z_{0}$ есть отталкивающая неподвижная точка $g_{02}, z_{1}$ есть отталкивающая неподвижная точка $g_{12}$.

В каждом из случаев существует многоугольник, удовлетворяющий вместе с $G$ условию леммы 2 . Применяя леммы 2 и 3 , получаем, что замыкание полугруппы $\bar{G}$ является группой.

Лемма 7 ([2]). Пусть $f, g$ - гиперболические изометрии, имеющие ровно одну общую неподвижную точку $z$. Тогда замыкание группь, порохсенной $f$ u $g$, содержит все параболические изометрии с неподвижной точкой $z$.

Лемма 8 ([1]). Пусть $f$ - әллиптическая изометрия бесконечного порядка, $g$ - изометрия, не фиксирующая неподвижную точку $f$. Тогда полугруппа, порождденная $f$ u g, всюду плотна в $S \Gamma$.

Применяя лемму 7 к $g_{01}$ и $g_{02}$, находим, что в $\bar{G}$ содержатся все параболические изометрии с неподвижной точкой $z_{0}$. Используя сопряжение $g_{10}$, получаем, что там также содержатся все параболические изометрии с неподвижной точкой $g_{10}\left(z_{0}\right) \neq z_{0}$. Из этого следует, что в $\bar{G}$ есть эллиптическая изометрия бесконечного порядка и по лемме $8 \bar{G}=S \Gamma$. Теорема доказана.

Автор благодарит Н.П. Долбилина за постановку задачи и многочисленные обсуждения результатов.

\section{СПИСОК ЛИТЕРАТУРЫ}

[1] Barany I., Beardon A. F. // Mathematika (London). 1996. V. 43. №1. P. 165-171. [2] Бердон А. Геометрия дискретных групп. М.: Наука, 1986.

Московский государственный университет им. М. В. Ломоносова E-mail: ordin@rnt.ru
Принято редколлегией 03.04.2000 